\title{
The Case Against Factorism: On the Labels of $\otimes$-Factor Hilbert-Spaces of Similar Particles in Quantum Mechanics
}

\section{F. A. Muller ${ }^{1,2}$ (D) Gijs Leegwater ${ }^{1}$}

Published online: 5 August 2020

(c) The Author(s) 2020

\begin{abstract}
We discuss the case against Factorism, which is the standard assumption in quantum mechanics that the labels of the $\otimes$-factor Hilbert-spaces in direct-product Hilbert-spaces of composite physical systems of similar particles refer to particles, either directly or descriptively. We distinguish different versions of Factorism and argue for their truth or falsehood. In particular, by introducing the concepts of snapshot Hilbert-space and Schrödingermovie, we demonstrate that there are Hilbert-spaces and $\otimes$-factorisations where the labels do refer, even descriptively, to similar particles, which renders them probabilistically absolutely discernible.
\end{abstract}

Keywords Elementary particles · Identical particles $\cdot$ Similar particles $\cdot$ Discernibility Quantum mechanics · Factorism · Distinguishability

\section{Factorism and Part States}

Consider $N \in \mathbb{N}_{+}$particles, labelled by numerals ' 1 ', '2', $\ldots$, ' $N$ '. In quantum mechanics (QM), every possible pure state of particle $j \in\{1,2, \ldots, N\}$ is mathematically represented by a Hilbert-vector of norm equal to 1 , up to a global phase factor $\left(\mathrm{e}^{\mathrm{i} \theta}, \theta \in[0,2 \pi)\right)$, belonging to Hilbert-space $\mathcal{H}_{j}$. The Hilbert-space $\mathcal{H}$ of a composite system $S^{N}$ of $N$ particles is (a subspace of) the direct-product Hilbert-space of the $N \otimes$-factor Hilbert-spaces:

$$
\mathcal{H} \sqsubseteq \mathcal{H}^{N}=\mathcal{H}_{1} \otimes \mathcal{H}_{2} \otimes \cdots \otimes \mathcal{H}_{N}
$$

A vector in $\mathcal{H}^{N}$ is symmetric iff it is invariant under every permutation of the labels, and anti-symmetric iff it is invariant under every even permutation, and invariant up to a

F. A. Muller

f.a.muller@esphil.eur.nl

http://www.staff.science.uu.nl/muller106/

Gijs Leegwater

g.leegwater@esphil.eur.nl

1 Erasmus School of Philosophy, Erasmus University Rotterdam, Woudestein, 3062 PA Rotterdam, The Netherlands

2 Descartes Centre for the History and Philosopy of Science, Faculty of Science, Utrecht University, Utrecht, The Netherlands 
factor -1 for every odd permutation; and similarly for statistical (or density) operators in $\mathcal{S}(\mathcal{H})$, which represent mixed states. According to the Symmetrisation Postulate of QM, for composite systems of similar fermions (i.e. having the same super-selected, or stateindependent, properties, like mass, charge, spin magnitude and baryon number), $\mathcal{H}$ is the anti-symmetric subspace of $\mathcal{H}^{N}$, denoted by $\mathcal{H}_{-}$, and for similar bosons, $\mathcal{H}$ is the symmetric subspace of $\mathcal{H}^{N}$, denoted by $\mathcal{H}_{+}$. All $\otimes$-factor Hilbert-spaces must then be chosen the same: $\mathcal{H}_{j}=\mathcal{H}_{k}$ for every $j, k \in\{1,2, \ldots, N\}$.

The permutation-operators act by definition on the labels of the $\otimes$-factor Hilbert-spaces, e.g. for $N=3$ and permutation 321 of 123 :

$$
\widehat{\Pi}_{321}|u\rangle_{1} \otimes|v\rangle_{2} \otimes|w\rangle_{3}=|w\rangle_{1} \otimes|v\rangle_{2} \otimes|u\rangle_{3} .
$$

One can suppress the labels in the vectors in (2) and let the typographical order do the labelling:

$$
\widehat{\Pi}_{321}|u\rangle \otimes|v\rangle \otimes|w\rangle=|w\rangle \otimes|v\rangle \otimes|u\rangle .
$$

Since $|u\rangle_{1},|w\rangle_{1} \in \mathcal{H}_{1},|v\rangle_{2}, \in \mathcal{H}_{2}$, and $|w\rangle_{3},|u\rangle_{3} \in \mathcal{H}_{3}$, and Hilbert-space $\mathcal{H}_{1}$ is associated with particle 1 , etc., what could be more natural than to assume that the labels also refer to the particles? Indeed, nothing could be more natural. In fact, we opened this article by introducing the labels as names of particles and then used them too as labels of the $\otimes$ -factor Hilbert-spaces. ${ }^{2}$ This assumption seems to have been first unearthed explicitly and turned into a subject of serious thought by Lubberdink in her ms-thesis of 1998, parts of which were published 13 years later with her supervisor Dieks; independently it was implicitly unearthed by Huggett and Imbo (2009), and essentially by Saunders in 2006; the assumption has been baptised insipidly Factorism by Caulton, first in his PhD-thesis of 2011. ${ }^{3}$ Insipidly, we follow suit:

Factorism. Given a Hilbert-space $\mathcal{H}$ of a composite system $\mathrm{S}^{N}$ of $N$ given similar parts, representing the possible physical states of $\mathrm{S}^{N}$, and given a direct-product factorisation of $\mathcal{H}$ into other Hilbert-spaces. Then the labels of these given $\otimes$-factor Hilbert-spaces refer to the $N$ parts either directly (Direct Factorism) or descriptively (Descriptive Factorism).

A number of systematic remarks on Factorism follow next.

First, we must realise that Factorism can only be judged true or false until and unless $\mathcal{H}$ has been specified and a $\otimes$-factorisation of $\mathcal{H}$ has been specified. When one uses symbols like ' $\mathcal{H}$ ' and ' $\mathcal{H}_{j}$ ', then nothing has been specified, because these are variables ranging over

\footnotetext{
1 Stricto sensu isomorphic: $\mathcal{H}_{j} \simeq \mathcal{H}_{k}$, for the $\otimes$-factors being isomorphic will also do, even when they are not identical; since all complex (separable) Hilbert-spaces of the same dimension are isomorphic, it is thus stricto sensu sufficient to require that the $\otimes$-factor Hilbert-spaces have the same dimension.

2 Standard in every textbook on QM and the overwhelming majority of papers on the subject of 'identical' (similar) particles. See e.g. Cohen-Tannoudji et al. (1978), Butterfield (1993), French and Redhead (1988), Redhead and Teller (1992), French and Krause (2006), Saunders (2006a, b), Muller and Saunders (2008), Muller and Seevinck (2009), Ladyman et al. (2012).

${ }^{3}$ Lubberdink (1998), Saunders (2006b), Huggett and Imbo (2009), Dieks and Lubberdink (2011), Caulton (2018b). Caulton currently prefers to call Direct Factorism "transcendental individuation", harking back to a terminology of Redhead c.s. Caulton (2018a), Redhead and Teller (1992), French and Redhead (1988). The terminology of 'transcendental individuation' should not lead us to believe that a metaphysical issue is at stake here: the interpretation of the $\times$-factor labels is a semantic issue.
} 
the class of all and only Hilbert-spaces. Then Factorism does not express a proposition that can be true or false. Factorism can be made independent of such specifications and then expressing a proposition, by quantifying the two-fold dependence away universally ( $\forall$-Factorism) or existentially ( $\exists$-Factorism), as follows:

$\forall$-Factorism. For every Hilbert-space $\mathcal{H}$ of a composite system $\mathrm{S}^{N}$ of $N$ given similar parts and for every direct-product factorisation of $\mathcal{H}$ into other Hilbert-spaces, the labels of these given $\otimes$-factor Hilbert-spaces refer to the $N$ parts either directly (Direct $\forall$-Factorism) or descriptively (Descriptive $\forall$-Factorism).

$\exists$-Factorism. For some Hilbert-space $\mathcal{H}$ of a composite system $\mathrm{S}^{N}$ of $N$ given similar parts and for some direct-product factorisation of $\mathcal{H}$ into other Hilbert-spaces, the labels of these given $\otimes$-factor Hilbert-spaces refer to the $N$ parts either directly (Direct $\exists$-Factorism) or descriptively (Descriptive $\exists$-Factorism).

It will be useful to define even a fourth thesis: ${ }^{4}$ :

Anti-Factorism. For every Hilbert-space $\mathcal{H}$ of a composite system $\mathrm{S}^{N}$ of $N$ given similar parts and every direct-product factorisation of $\mathcal{H}$ into other Hilbert-spaces, the labels of these given $\otimes$-factor Hilbert-spaces neither refer to the $N$ parts directly (Direct Factorism) nor descriptively (Descriptive Factorism).

These three theses express distinct propositions, which are logically related. If $\forall$-Factorism is true, then $\exists$-Factorism is true, and Anti-Factorism is false. If $\exists$-Factorism is true, then nothing follows about $\forall$-Factorism, it can be true and it can be false; but then AntiFactorism again is false. If $\forall$-Factorism is false, then both $\exists$-Factorism and Anti-Factorism can be true or can be false. If Anti-Factorism is true, then both $\forall$-Factorism and $\exists$-Factorism are false. If Anti-Factorism is false, then $\exists$-Factorism is true and $\forall$-Factorism can be true or false. It will transpire that $\exists$-Factorism is true, $\forall$-Factorism is false, and hence AntiFactorism is also false.

Notice that for composite systems of dissimilar parts, the labels of the $\otimes$-factors do refer unproblematically to these dissimilar parts. Which is why all versions of Factorism mention explicitly that the parts must be similar.

Secondly, it is rarely clear which version of Factorism is criticised by the authors listed above. Their generic use of symbols like ' $\mathcal{H}$ ' and ' $\mathcal{H}$ ' suggest they talk about an arbitrary Hilbert-space, which makes it logically equivalent to talking about all Hilbert-space (by the introduction-rule of ' $\forall$ '). This would be Anti-Factorism and therefore they seemed to believe to have established the truth of Anti-Factorism. The context however usually reveals they have been considering a specific Hilbert-space, notably Dieks and Lubberdink (2011), and a specific $\otimes$-factorisation, which means that all they have established is that there is a Hilbert-space and there is a $\otimes$-factorisation of which the labels do not refer to the particles, in other words, they have established that $\forall$-Factorism is false by producing a counter-example. This leaves the truth of both $\exists$-Factorism and Anti-Factorism wide open. Again, we shall conclude the falsehood of Anti-Factorism and thereby the truth of $\exists$ -Factorism.

\footnotetext{
4 The name of which has also been introduced by Caulton $(2012, \S 2.2)$ : "Anti-factorism is the doctrine that factor Hilbert-space indices are a gauge quantity." These labels "do not represent anything physical". When Caulton means here the $\otimes$-factor Hilbert-spaces of all $\otimes$-factorisations of all Hilbert-spaces, then this doctrine is the same as our Anti-Factorism.
} 
Thirdly, we must also realise that every version of Factorism is an assumption, because it does not follow from 'the mathematics' or the Von Neumann postulates of QM. Like postulating that certain Hilbert-vectors represent physical states and certain operators represent physical magnitudes, Factorism is an assumption that tells us that certain mathematical entities, the labels (for which standardly positive natural numbers are used), besides naming $\otimes$-factor Hilbert-spaces and naming operators acting in them (generically operator $A_{j}$ acting in $\mathcal{H}_{j}$ ), are also names of the particles. Physical significance is attributed to these labels - and explicity denied by Anti-Factorism.

Fourthly, as Friebe has pointed out, connecting Factorism to the philosophy of language, that standardly the labels refer directly to the particles, they are senseless names, in contrast to descriptive names, which refer descriptively by Russellian definite descriptions. ${ }^{5}$ Whence the difference between Direct and Descriptive Factorism. In order to refer descriptively to a constituent particle of a physical system, the particle must be absolutely discerned from the other constituent particles, which means it must possess at least one property that all other particles lack. In the case under consideration, of physical systems composed of similar particles, descriptive reference seems impossible because all constituent particles share all their properties and seem therefore absolutely indiscernible; direct reference is then the only available option to refer. Of course, one can discern the particles absolutely by means of labels (the particle having label ' 1 '), but since these senseless labels lack any physical significance, such discernment is physically insignificant. The particles remain physically, or empirically, absolutely indiscernible. (In fact, what is permitted to discern and what is forbidden to discern in the context of QM-and beyond-has recently been scrutinised. ${ }^{6}$ ) When probability measures occur in the relations, one speaks of probabilistic discernment.

Do the constituent parts of a composite physical system generally also have states? Call the answer in the affirmative:

Part States. All parts of a composite physical system have states.

As soon as an answer in the affirmative has been given, the next question is: what is the state of the parts, and how does it relate to the state of the composite system? Are the states of similar particles different? Before we address these questions, let us first see what arguments are around in favour of adopting Part States.

The most general conception of a physical state is the mixed one, as Von Neumann proposed, and mixed states are represented by statistical operators in the convex set $\mathcal{S}\left(\mathcal{H}_{ \pm}\right)$ (positive, self-adjoint, trace class1); the expectation-value of a physical magnitude represented by symmetric operator $A$ when the physical system is in state $W$ is provided by the celebrated trace-formula:

$$
\langle A\rangle_{W}=\operatorname{Tr}(A W) .
$$

By choosing for $A$ the members of its spectral resolution, formula (4) yields a probabilitymeasure for $A$-measurement outcomes.

1. One reason in favour of Part States is that Part States needs no reason in QM because in QM it follows from the State Postulate of QM: (i) the members of $\mathcal{S}(\mathcal{H})$ represent the possible physical states of a physical system with which $\mathcal{H}$ is associated, and (ii) the

\footnotetext{
${ }^{5}$ See Friebe (2014). To wit, Friebe defends descriptivism but not Descriptive Factorism.

${ }^{6}$ Muller and Saunders (2008), Muller and Seevinck (2009), Muller (2015).
} 
members of $\mathcal{S}\left(\mathcal{H}_{1} \otimes \mathcal{H}_{2} \otimes \cdots \mathcal{H}_{N}\right)$ represent those of a composite system of $N$ parts. Since the parts of the last-mentioned physical system are physical systems too, with which the $\otimes$-factor Hilbert-spaces are associated, it follows from (i) that the possible states of part $j$ are represented by members of $\mathcal{S}\left(\mathcal{H}_{j}\right)$. Yet one may weaken the State Postulate to (ii), and leave it open whether parts have states too, or even deny that parts have states. In that case, Part States does not follow from the State Postulate, and we may now legitimately request a reason for Part States.

2. The standard reason for Part States goes as follows. In principle we can perform a measurement of any physical magnitude pertaining to a part, viz. the position of a single particle, represented by a symmetrised one-particle position-operator. Repeated measurements test a single-particle probability-measure. Since probability-measures of physical magnitudes pertaining to the composite system are generated by some statistical operator $W \in \mathcal{S}\left(\mathcal{H}_{ \pm}\right)$and some symmetric operator $A$ acting in $\mathcal{H}_{ \pm}$( $W$ is guaranteed to exist by Gleason's Theorem), we also want single-particle probability-measures to be generated by some statistical operator $W_{j} \in \mathcal{S}\left(\mathcal{H}_{j}\right)$ and some operator $A_{j}$ acting in $\mathcal{H}_{j}\left(W_{j}\right.$ is also guaranteed to exist by Gleason's Theorem). But which one? As Van Fraassen has pointed out, all we need to obtain Von Neumann's choice that $W_{j}$ is the partial trace of $W$ is to endorse the eminently reasonable requirement that the expectation-value of $A_{j}$ acting in $\mathcal{H}_{j}$ when particle $j$ is in state $W_{j}$ equals the one of the trivially extended $A_{j}$ to act symmetrically in $\mathcal{H}_{ \pm}$ when the composite system is in state $W \in \mathcal{S}\left(\mathcal{H}_{ \pm}\right):^{7}$

$$
\operatorname{Tr}\left(A_{j} W_{j}\right)=\operatorname{Tr}(A W) .
$$

For two particles, $A$ is $\frac{1}{2}\left(A_{1} \otimes I_{2}+I_{1} \otimes A_{2}\right)$, etc.

Notice that in both reasons, $\forall$-Factorism seems to have been taken for granted.

Next it is elementary to show that the partial traces of similar fermions are identical, and for similar bosons too, so that the single-particle states cannot be used to discern the particles absolutely, and hence cannot be used to refer to the particles descriptively in what would have been a physically significant manner. ${ }^{8}$ For fermions, the states are always improper mixtures; for bosons, pure states and improper mixtures are possible, never proper mixtures. Thus the similar particles remain absolutely indiscernible, even after Part States has been embraced. They cannot be individuated and therefore are not individuals; they are indiscernibles. The adoption of Part States has brought no change in the situation that according to QM, similar particles are indiscernibles. Moreover, Direct Factorism is the name of the reference game of similar particles in QM. Descriptive Factorism is out.

In order to prevent that an operator representing a physical magnitude takes Hilbertvectors outside $\mathcal{H}_{ \pm}$, it must be symmetric, which is to say that it must commute with every permutation operator. This is known as the Indistinguishability Postulate, which is not a postulate because it follows from the Symmetrisation Postulate. ${ }^{9}$ That symmetrised operators must be used when considering similar particles is well-known. Less well-known is that when we want to measure a physical magnitude pertaining to a single particle, one must distinguish, in case of two particles, between (i) whether a single particle has a

\footnotetext{
7 Fraassen (1991, 199).

8 Fraassen (1992, 386), QM-Exclusion Corollary.

9 But notoriously not conversely. See Fraassen (1992, 381 ff), Huggett and Imbo (2009, § 3).
} 
property and the other does not have it, and (ii) whether at least one particle has it. Bigaj (2015b, 193-195) explains how and why.

Some have argued that the similar particles can be discerned by relations in physically significant ways, which turns the similar particles into relationals, in contrast to indiscernibles and individuals. ${ }^{10}$ The first criticism against this relational discernment was that it is circular; this criticism has not stood up to scrutiny. ${ }^{11}$ The second criticism is that the discerning relations in some instances employ operators that do not obey the Indistinguishability Postulate; hence no physical significance is bestowed on the discerning relations by the operators employed in defining these relations. ${ }^{12}$ Repair jobs of relational discernment have been mounted; some repair jobs have been succesful for some but not all classes of states, another repair job has been succesful for all states but is probabilistic in nature. ${ }^{13}$ Leibniz's Principle of the Identity of Indiscernibles is a metaphysical principle that is at stake in this debate. The proponents of relational discernibility have argued that the sufficient condition for identity of sharing all properties must be extended to include also relations, in which case the principle is obeyed rather than violated by QM. The debate about relational discernibility and Leibniz's principle continues but we park it here. For even if similar particles are relationals, they are not individuals, not absolutely discernible, and Direct Factorism then seems the only manner to refer to the particles.

We next turn to the arguments against Factorism (Sects. 2, 5), which we shall counter (Sects. 3, 5). Further, we mention a few things that are just non-trivial enough to be worthy to be pointed out at all (Sect. 4, which can be omitted) and we end with concluding remarks (Sect. 6).

\section{Factorisable, Tangled and Entangled States}

Consider a composite system of a proton and an electron with Hilbert-spaces $\mathcal{H}_{1}=L^{2}\left(\mathbb{R}^{3}\right)$ and $\mathcal{H}_{2}=\mathbb{C}^{2}$ (which is admittedly a peculiar combination). This system can be in some product-state:

$$
|\psi\rangle \otimes\left|\uparrow_{z}\right\rangle=\psi \otimes\left(\begin{array}{l}
1 \\
0
\end{array}\right) \in L^{2}\left(\mathbb{R}^{3}\right) \otimes \mathbb{C}^{2} .
$$

The particle labelled ' 1 ' can now be descriptively referred to as 'the particle having stateindependent properties mass $m_{\mathrm{p}}$, charge +1 and spin-magnitude 1/2', and mutatis mutandis for '2'. The particles can also be descriptively referred to by their states: particle1 is the particle in the state represented by wave-function $\psi$, and particle 2 is the particle in the state represented by the column-vector in (6). In superpositions, which prohibit the ascription of properties corresponding to magnitudes whose eigenvectors are members of the basis in which the superposition is expressed, neither state-dependent properties nor states of parts can be used for descriptive reference. But the state-independent properties can always be used to do the job of descriptive reference. These particles are absolutely discernible, they can be discerned in physically significant ways, they are individuals, and

\footnotetext{
10 Saunders (2006a, b), Muller and Saunders (2008), Muller and Seevinck (2009), Saunders (2013).

11 French and Krause (2006), Hawley (2009), Muller (2015).

12 Huggett and Norton (2014), Caulton (2013), Bigaj (2015a).

13 See references in the previous footnote, and Friebe (2014).
} 
they can be individuated by their state-independent properties, which they always possess. For composite systems of dissimilar particles, Descriptive Factorism holds good. Direct Factorism holds good too, but has become otiose.

There are three arguments against Factorism around, which all pertain to composite systems of similar particles:

- The Argument from Tangled States (current section).

- The Argument from Entangled States (current section).

- The Argument from Measurement (Sect. 5).

Before we exposition these arguments, first some preparations.

Since similar particles cannot be discerned by: their state-independent properties, by their physically insignificant senseless labels, or by their states if they have them (their states are all the same), the only way to discern them is by means of their state-dependent properties (or relations). Here the troubles begin. Product-states, like $|u\rangle_{1} \otimes|v\rangle_{2}$, cannot be anti-symmetric, so turning the directly referring labels into descriptive ones by means of the states is not on. ${ }^{14}$ Product-states can be symmetric, but only when the $\otimes$-factors are alike, as in: $|u\rangle_{1} \otimes|u\rangle_{2}$, which means that both particles are in exactly the same state $|u\rangle$ and hence discernment by means of the states is not on.

The inquiries into entanglement by Ghirardi et al. (2002) c.s. has however led to the view that there are non-factorisable states in which particles can be discerned by means of descriptive reference. ${ }^{15}$ They call non-factorisable states that result from anti-symmetrising a product-state 'not entangled'; we shall call them tangled. Call a non-factorisable state that does not result from (anti-)symmetrising some product-state entangled. The non-factorisable vectors then subdivide in tangled and entangled ones. One good reason to draw this distinction in the non-factorisable states is that only entangled states, when considering spin and position, lead to a breach of a Bell-inequality, whereas tangled states do not lead to a breach. ${ }^{16}$ When in 1935 , Schrödinger famously wrote about what he called 'entanglement': "I would not call that one but rather the characteristic trait of quantum mechanics, the one that enforces its entire departure from classical lines of thought", one can only endorse this when by 'entanglement' is meant how Girardi et al. characterised it, to the exclusion of the non-factorisable tangled states, which do not warrant a departure from classical lines of thought. (But see the Envoi in Sect. 7.)

For the sake of clarity and future reference, here are three running examples of Hilbertvectors of each type, for two similar spin 1/2-fermions, with Pauli-spinor Hilbert-space $L^{2}\left(\mathbb{R}^{2}\right)^{2}$ as the single-particle state-space (thus including all degrees of freedom: position, momentum, spin):

\footnotetext{
${ }^{14}$ If $|u\rangle_{1} \otimes|v\rangle_{2}=\Pi_{12}|u\rangle_{1} \otimes|v\rangle_{2}=-|v\rangle_{1} \otimes|u\rangle_{2}$, then $|v\rangle$ or $|u\rangle$, or both, must be the zero vector; but the zero vector does never represent a physical state.

${ }^{15}$ Ghirardi et al. (2002), Ghirardi and Marinatto (2004), Ladyman et al. (2013), Friebe (2014).

${ }^{16}$ Gisin (1991) proved that all non-factorisable states of two dissimilar particles give rise to a breach of Bell's inequality. For the different case of similar particles, see e.g. Caulton (2018a), Theorem 3.4, Corollary 4.4.
} 


$$
\begin{array}{ll}
\text { factorisable } & :|\Phi(t)\rangle=\left|L, \uparrow_{z} ; t\right\rangle \otimes\left|R, \downarrow_{z} ; t\right\rangle . \\
\text { tangled } & :|\Theta(t)\rangle=\frac{1}{\sqrt{2}}\left(\left|L, \uparrow_{z} ; t\right\rangle \otimes\left|R, \downarrow_{z} ; t\right\rangle-\left|R, \downarrow_{z} ; t\right\rangle \otimes\left|L, \uparrow_{z} ; t\right\rangle\right) . \\
\text { entangled } & :|\Psi(t)\rangle=\frac{1}{2}\left(\left|L, \uparrow_{z} ; t\right\rangle \otimes\left|R, \downarrow_{z} ; t\right\rangle-\left|L, \downarrow_{z} ; t\right\rangle \otimes\left|R, \uparrow_{z} ; t\right\rangle\right. \\
& \left.+\left|R, \uparrow_{z} ; t\right\rangle \otimes\left|L, \downarrow_{z} ; t\right\rangle-\left|R, \downarrow_{z} ; t\right\rangle \otimes\left|L, \uparrow_{z} ; t\right\rangle\right) .
\end{array}
$$

Wave functions $|L ; t\rangle,|R ; t\rangle \in L^{2}\left(\mathbb{R}^{3}\right)$ are wave-packets moving in opposite directions (called 'Left' and 'Right') in configuration space $\mathbb{R}^{3}$ :

$$
R(\mathbf{q} ; t)=\frac{1}{(2 \pi)^{3 / 2}} \int_{\mathbb{R}^{3}} A(\mathbf{k}, 0) \exp (\mathrm{i}(\mathbf{k} \cdot \mathbf{q}-\omega(k) t)) d \mathbf{k} \in L^{2}\left(\mathbb{R}^{3}\right),
$$

and

$$
L(\mathbf{q} ; t)=\frac{1}{(2 \pi)^{3 / 2}} \int_{\mathbb{R}^{3}} A(\mathbf{k}, 0) \exp (\mathrm{i}(\mathbf{k} \cdot \mathbf{q}+\omega(k) t)) d \mathbf{k} \in L^{2}\left(\mathbb{R}^{3}\right),
$$

with dispersion function $\omega(k)=\hbar k^{2} / 2 m$, wave-vector $\mathbf{k}=\mathbf{p} / \hbar=m \mathbf{v} / \hbar$, and length $k=\|\mathbf{k}\|$. Velocity $\mathbf{v}$ is the velocity of the peak of the wave-packet in configuration-spacenot the velocity of the particle. Here are the Pauli-spinors:

$$
\left|L, \uparrow_{z} ; t\right\rangle=\left(\begin{array}{c}
L(t) \\
0
\end{array}\right) \text { and }\left|L, \downarrow_{z} ; t\right\rangle=\left(\begin{array}{c}
0 \\
L(t)
\end{array}\right),
$$

and mutatis mutandis for $\left|R, \uparrow_{z} ; t\right\rangle$ and $\left|R, \downarrow_{z} ; t\right\rangle$.

The Argument against Descriptive Factorism from Tangled States. As we mentioned above, factorisable vectors like $|\Phi(t)\rangle$ do not represent possible physical states of a similar particles system. Ghirardi c.s. submit that the tangled vector $|\Theta(t)\rangle$ represents a state such that one particle has $z$-spin up and is moving to the left (Left- $z \mathrm{Up}$ ) and the other particle has $z$-spin down and is moving to the right (Right-zDown). ${ }^{17}$ For dissimilar particles, such a state is represented by $|\Phi(t)\rangle$, but the Symmetrisation Postulate forbids this and makes us anti-symmetrise $|\Phi(t)\rangle$, which yields $|\Theta(t)\rangle$. This interpretation of $|\Theta(t)\rangle$ contravenes the standard Strong Property Postulate (aka under the somewhat misleading name 'EigenstateEigenvalue Link $\left.{ }^{18}\right)$, according to which a physical system in pure state $|\psi\rangle$ has quantitative property $\langle A, a\rangle$ iff $A|\psi\rangle=a|\psi\rangle .{ }^{19}$ Hilbert-vector $|\Theta(t)\rangle$ is neither in an eigenstate of any spectral projector of the position-operator (wave-packets have infinite tails) nor in an eigenstate of $\sigma_{z}$, and then can neither be localised in any spatial region nor possess a $z$-spin property $\left\langle\sigma_{z}, \pm 1\right\rangle$. Particles can have quantitative properties even when not in eigenstates, say Ghirardi et al., provided their non-factorisable state is tangled. Descriptive reference becomes a viable option again. ${ }^{20}$

\footnotetext{
17 Ghirardi et al. (2002, 86).

18 Misleading, because the eigenvalue-eq. connects eigenstates to eigenvalues, which is a mathematical connexion that does not involve properties. The Strong Poperty Postulate states a strong connexion between a physical system possessing quantitative properties and having a particular type of state.

19 Formulations encompassing continuous spectra and mixed states are available but glossed over.

20 About 25 years ago, Van Fraassen also presented, in the context of the modal interpretation of QM (which also renounces the Strong Property Postulate), a model of a composite system of fermions with individuation, that is, with descriptive reference, turning the fermions into absolutely discernible objects. Fraassen (1992, 427 ff.)
} 
For the Factorist who wants to endorse this interpretation, the following problem arises. Was the particle initially directly referred to by label ' 1 ' Left- $z$ Up or Right- $z$ Down? The Factorist cannot answer: it has to be one of them, because we all agree there are two particles. The descriptions Left- $z$ Up and Right- $z$ Down are in the tangled state associated to two vectors, each one in a different Hilbert-space, and therefore associated to both labels ' 1 ', and 2 '.

So it seems there is strong case that for tangled states, the Hilbert-space labels do not refer to particles, and for those states, Direct as well as Descriptive Factorism fails.

The Argument against Factorism from Entangled States. The move to descriptive reference is unavailable for the entangled state $|\Psi(t)\rangle(7)$, which is empirically distinguishable from the tangled state $|\Theta(t)\rangle$, for there is no correlation anymore in the terms between spin and space. Both Alice (at the left wing of the celebrated Einstein-Podolsky-Rosen-Bohm-Bell experiment) and Bob (at the right wing) have a probability of $1 / 2$ to find $z$-spin up and to find $z$-spin down, whereas in the tangled state $|\Theta(t)\rangle$, Alice has nearly probability equal to 1 to find spin up (Left- $z \mathrm{Up}$ ) and equally Bob to find spin down (Right-zDown). ${ }^{21}$ Spin and space now have become entangled. Direct reference returns. One can maintain that the Hilbert-space labels refer to the particles and that these senseless names cannot be filled with physical significance by means of physically significant descriptions as in the tangled case. Descriptive Factorism is not on. Yet this implies that Direct Factorism with its senseless, referring labels remains the only live option to refer.

But perhaps descriptive reference is on after all. When in the tangled state $|\Theta(t)\rangle$ we can have Left- $z$ Up and Right- $z$ Down, why can we not have in the entangled state $|\Psi(t)\rangle$ Leftand-no-z-spin-property and Right-and-no-z-spin-property? We can have that.

We can calculate the probability that Alice and Bob find a particle in their detector, while not measuring its spin; they then perform a position measurement. Here are the Born position-probability densities, which 'move' in ordinary space (represented by $\mathbb{R}^{3}$ ); one is moving towards Alice:

$$
\rho_{L}(\mathbf{r}, t) \equiv 1_{\{\mathbf{r}\}}\left(\mathbf{q}_{1}\right)\left|L\left(\mathbf{q}_{1} ; t\right)\right|^{2}+1_{\{\mathbf{r}\}}\left(\mathbf{q}_{2}\right)\left|L\left(\mathbf{q}_{2} ; t\right)\right|^{2}=2|L(\mathbf{r}, t)|^{2},
$$

and the other one is moving towards Bob:

$$
\rho_{R}(\mathbf{r}, t) \equiv 1_{\{\mathbf{r}\}}\left(\mathbf{q}_{1}\right)\left|R\left(\mathbf{q}_{1}, t\right)\right|^{2}+1_{\{\mathbf{r}\}}\left(\mathbf{q}_{2}\right)\left|R\left(\mathbf{q}_{2}, t\right)\right|^{2}=2|R(\mathbf{r}, t)|^{2},
$$

where $1_{\{\mathbf{r}\}}$ is the characteristic function of singleton-set $\{\mathbf{r}\}$, so that $1_{\{\mathbf{r}\}}(\mathbf{q})=1$ if $\mathbf{q}=\mathbf{r}$, and $1_{\{\mathbf{r}\}}(\mathbf{q})=0$ if $\mathbf{q} \neq \mathbf{r} .^{22}$ We have again two absolutely discernible particles, discerned by their distinct position-probability densities, $\rho_{L}$ and $\rho_{R}$, respectively. The particles have become individuals.

Dieks and Lubberdink (2011) have indicated that this way of looking at entangled states-and tangled states, if not all states whenever warranted-is needed for the classical limit of QM. The peaks of the position-probabilities in space, provided we end up with $N$ single-peaked position-probabilities in space for $N$-particle systems, follow classical trajectories according to Ehrenfest's Theorem, because the peaks move with a velocity in space equal the expectation-value of the velocity-operator in such cases.

\footnotetext{
21 Nearly, because the position probabilities have infinite tails.

22 These position-probability densities can also be obtained from the ones in configuration space by means of delta-distributions: $\delta\left(\mathbf{q}_{1}-\mathbf{r}\right)$ and $\delta\left(\mathbf{q}_{2}-\mathbf{r}\right)$.
} 
The situation with the entangled state thus has become the same as with the tangled state. Both position probabililty-densities involve both labels, manifest in (11) and in (12) by ' $\mathbf{q}_{1}$ ' and ' $\mathbf{q}_{2}$ '. So just as in the tangled case, the Hilbert-space labels do not refer to particles. Direct as well as Descriptive Factorism fails.

There is however one caveat. The wave-packets of non-interacting particles, as in the EPRB-case, spread rather quickly. Within no time, the position-probability densities are nearly uniform. For example, when we consider electrons and a Gaussian wave-packet having a width equal to the Bohr radius (the size of an $\mathrm{H}$-atom in its ground state: $10^{-11} \mathrm{~m}$ ), then after one second, the width has become:

$$
\Delta x(t)=\frac{a}{2} \sqrt{1+\frac{4 \hbar^{2}}{m_{\mathrm{e}}^{2} a^{4}} t^{2}} \approx 10^{-11} \sqrt{1+\frac{\left(10^{-34}\right)^{2}}{\left(10^{-30}\right)^{2}\left(10^{-11}\right)^{4}}} \approx 10^{6} \mathrm{~km},
$$

which is about three times the Lunar distance. The probability to find the electron at $1 \mathrm{sec}-$ ond beyond the moon is $1 / 3$. The situation of absolute discernment is over in the wink of an eye.

Cold comfort for the Factorist, because a situation of nearly uniform wave-packets is not going to help Factorism to regain its long reign of dominance, albeit shifting Hilbertspaces. So Factorism rest in peace after all? Not so quick. All considerations above are about a specified Hilbert-space, the anti-symmetric subspace of infinite-dimensional Paulispinor Hilbert-space:

$$
\left(L^{2}\left(\mathbb{R}^{3}\right)^{2} \otimes L^{2}\left(\mathbb{R}^{3}\right)^{2}\right)_{-}
$$

But what if we specify a different Hilbert-space? We shall see what then happens in the next section.

\section{Snapshot Hilbert-Space and Schrödinger-Movies}

We can bury $\forall$-Factorism with appropriate honours, that much should be evident by now. But how about $\exists$-Factorism?

We now construct a 4-dimensional proper subspace of Pauli-spinor space (14) with descriptively referring $\otimes$-factor Hilbert-space labels. During the short time-interval where the criticism of Factorism has bite, the pairs of wave-functions are approximately orthogonal:

$$
\langle L ; t \mid R ; t\rangle=\int_{\mathbb{R}^{3}} L^{*}(\mathbf{q} ; t) R(\mathbf{q} ; t) d \mathbf{q} \approx 0
$$

Now we consider the direct-product Hilbert-space spanned by the following tangled vectors: ${ }^{23}$

\footnotetext{
${ }^{23}$ A similar contruction is alluded to by Huggett and Imbo (2009), Section 2, eq. (3). Caulton (2018a) also takes heed, below Corollary 4.4.
} 


$$
\begin{aligned}
& \left|\uparrow_{z} \uparrow_{z} ; t\right\rangle \equiv \frac{1}{\sqrt{2}}\left(\left|L, \uparrow_{z} ; t\right\rangle \otimes\left|R, \uparrow_{z} ; t\right\rangle-\left|R, \uparrow_{z} ; t\right\rangle \otimes\left|L, \uparrow_{z} ; t\right\rangle\right), \\
& \left|\uparrow_{z} \downarrow_{z} ; t\right\rangle \equiv \frac{1}{\sqrt{2}}\left(\left|L, \uparrow_{z} ; t\right\rangle \otimes\left|R, \downarrow_{z} ; t\right\rangle-\left|R, \downarrow_{z} ; t\right\rangle \otimes\left|L, \uparrow_{z} ; t\right\rangle\right), \\
& \left|\downarrow_{z} \uparrow_{z} ; t\right\rangle \equiv \frac{1}{\sqrt{2}}\left(\left|L, \downarrow_{z} ; t\right\rangle \otimes\left|R, \uparrow_{z} ; t\right\rangle-\left|R, \uparrow_{z} ; t\right\rangle \otimes\left|L, \downarrow_{z} ; t\right\rangle\right), \\
& \left|\downarrow_{z} \downarrow_{z} ; t\right\rangle \equiv \frac{1}{\sqrt{2}}\left(\left|L, \downarrow_{z} ; t\right\rangle \otimes\left|R, \downarrow_{z} ; t\right\rangle-\left|R, \downarrow_{z} ; t\right\rangle \otimes\left|L, \downarrow_{z} ; t\right\rangle\right) .
\end{aligned}
$$

We $\otimes$-factorise this snapshot Hilbert-space $\mathcal{H}(t)$ into $\mathcal{H}_{1}(t)$ and $\mathcal{H}_{\mathrm{r}}(t)$ such that:

$$
\left|\uparrow_{z} \uparrow_{z} ; t\right\rangle=\left|\uparrow_{z} ; t\right\rangle_{1} \otimes\left|\uparrow_{z} ; t\right\rangle_{\mathrm{r}}, \quad\left|\uparrow_{z} \downarrow_{z} ; t\right\rangle=\left|\uparrow_{z} ; t\right\rangle_{1} \otimes\left|\downarrow_{z} ; t\right\rangle_{\mathrm{r}}, \quad \text { etc., }
$$

where $\left|\uparrow_{z} ; t\right\rangle_{1} \in \mathcal{H}_{1}(t)$ and $\left|\uparrow_{z} ; t\right\rangle_{\mathrm{r}} \in \mathcal{H}_{\mathrm{r}}(t)$. Then we have:

$$
\mathcal{H}(t)=\mathcal{H}_{1}(t) \otimes \mathcal{H}_{\mathrm{r}}(t) \sqsubset L^{2}\left(\mathbb{R}^{6}\right)^{4} .
$$

The history of the two-fermion system is some curve in $L^{2}\left(\mathbb{R}^{6}\right)^{4}$. The continuous sequence of 4-dimensional snapshot Hilbert-spaces $t \mapsto \mathcal{H}_{1}(t) \otimes \mathcal{H}_{\mathrm{r}}(t)$, which we call the Schrödinger-movie, envelops this history. ${ }^{24}$ In this movie, the single-particle labels ' 1 ' and ' $r$ ' can be taken as descriptive names: particle 1 is the particle moving to the left in space (Left) and $\mathrm{r}$ is the particle moving to the right in space (Right), but neither particle possesses a $z$-spin property $\left\langle\sigma_{z}, \pm\right\rangle .{ }^{25}$ So when it said that we are dealing with 'two particles in the singlet state moving in opposite directions', then this description does not fit the Bohm singlet state:

$$
\frac{1}{\sqrt{2}}\left(\left|\uparrow_{z}\right\rangle \otimes\left|\downarrow_{z}\right\rangle-\left|\downarrow_{z}\right\rangle \otimes\left|\uparrow_{z}\right\rangle\right) \in \mathbb{C}^{2} \otimes \mathbb{C}^{2}=\mathbb{C}^{4},
$$

because the spatial degrees of freedom are absent from (19), but these words do fit the spin-space entangled state $|\Psi(t)\rangle(7)$ in the snapshot basis:

$$
|\Psi(t)\rangle=\frac{1}{\sqrt{2}}\left(\left|\uparrow_{z} \downarrow_{z} ; t\right\rangle-\left|\downarrow_{z} \uparrow_{z} ; t\right\rangle\right)=\frac{1}{\sqrt{2}}\left(\left|\uparrow_{z} ; t\right\rangle_{1} \otimes\left|\downarrow_{z} ; t\right\rangle_{\mathrm{r}}-\left|\downarrow_{z} ; t\right\rangle_{1} \otimes\left|\uparrow_{z} ; t\right\rangle_{\mathrm{r}}\right) .
$$

When we watch the tangled $|\Theta(t)\rangle$ in the Schrödinger-movie, it factorises:

$$
|\Theta(t)\rangle==\left|\uparrow_{z} \downarrow_{z} ; t\right\rangle=\left|\uparrow_{z} ; t\right\rangle_{1} \otimes\left|\downarrow_{z} ; t\right\rangle_{\mathrm{r}},
$$

which has single-particle Hilbert-space labels that we take to refer descriptively: 1 is Left$z \mathrm{Up}$ and $\mathrm{r}$ is Right- $z$ Down.

So neither the argument against Factorism from Tangled States nor the one from Engtangled States is as decisive as their proponents believe: they wound $\forall$-Factorism lethally, but leave $\exists$-Factorism scatheless. In fact, for the case of two spin-1/2 fermions, we have proved $\exists$-Factorism.

Before we turn to the third and last argument against Factorism, we first want to make a few remarks inspired by our Schrödinger-movie.

\footnotetext{
24 This history is a solution of Schrödinger's equation, whence the name.

25 The spatial meaning of the labels makes this come close to Friebe's (2014) Kantian irreducible demonstratives.
} 


\section{Intermezzo: Parts and Degrees of Freedom}

This section contains a few explanatory remarks about QM in general, which can be passed over. An arbitrary vector in the Pauli-spinor Hilbert-space of one electron can be expanded based on basis-vectors $\alpha_{j} \in L^{2}\left(\mathbb{R}^{3}\right)\left(j \in \mathbb{N}_{+}\right)$:

$$
\left(\begin{array}{c}
\phi \\
\psi
\end{array}\right)=\sum_{j=1}^{\infty}\left(\left\langle\alpha_{j} \mid \phi\right\rangle\left(\begin{array}{c}
\alpha_{j} \\
0
\end{array}\right)+\left\langle\alpha_{j} \mid \psi\right\rangle\left(\begin{array}{c}
0 \\
\alpha_{j}
\end{array}\right)\right) .
$$

When we now write:

$$
\left(\begin{array}{c}
\alpha_{j} \\
0
\end{array}\right)=\alpha_{j} \otimes\left(\begin{array}{l}
1 \\
0
\end{array}\right) \quad \text { and } \quad\left(\begin{array}{c}
0 \\
\alpha_{j}
\end{array}\right)=\alpha_{j} \otimes\left(\begin{array}{l}
0 \\
1
\end{array}\right),
$$

it is obvious that we can write Pauli-spinor space as the direct product $L^{2}\left(\mathbb{R}^{2}\right) \otimes \mathbb{C}^{2}$. This shows that the physical significance of ' $\otimes$ ' is ambiguous. Usually it marks the combination of Hilbert-spaces pertaining to the different subsystems of a composite system. But here we have a single electron, which has no parts. In this case, ' $\otimes$ ' marks a separation of (spatial and spin) degrees of freedom.

For two spin-1/2 particles, we then can write (the anti-symmetric subspace of) Paulispinor Hilbert-space (14) as follows:

$$
\left(L^{2}\left(\mathbb{R}^{3}\right)^{2} \otimes L^{2}\left(\mathbb{R}^{3}\right)^{2}\right)_{-} \simeq\left(L^{2}\left(\mathbb{R}^{3}\right) \otimes L^{2}\left(\mathbb{R}^{3}\right) \otimes \mathbb{C}^{2} \otimes \mathbb{C}^{2}\right)_{-} \simeq\left(L^{2}\left(\mathbb{R}^{6}\right) \otimes \mathbb{C}^{4}\right)_{-} .
$$

The physical significance of ' $\otimes$ ' in the left expression is one of different composing parts, in the right expression one of separation of different degrees of freedom, and in the middle expression both kinds of physical significance occur.

Another thing to notice about ' $\otimes$ ' is that $\otimes$-factorisations are not unique, which has as a consequence that by staring at a $\otimes$-factorised Hilbert-space, we will not get a clue what the modelled physical system is and even not about how many particles compose it. For example, we have:

$$
\begin{aligned}
L^{2}\left(\mathbb{R}^{6}\right) & \simeq L^{2}\left(\mathbb{R}^{3}\right) \otimes L^{2}\left(\mathbb{R}^{3}\right) \\
& \simeq L^{2}\left(\mathbb{R}^{2}\right) \otimes L^{2}\left(\mathbb{R}^{2}\right) \otimes L^{2}\left(\mathbb{R}^{2}\right) \\
& \simeq L^{2}(\mathbb{R}) \otimes L^{2}(\mathbb{R}) \otimes L^{2}(\mathbb{R}) \otimes L^{2}(\mathbb{R}) \otimes L^{2}(\mathbb{R}) \otimes L^{2}(\mathbb{R})
\end{aligned}
$$

which is a state space of two particles in 3 spatial dimensions, and of three particles in the plane $(\mathbb{R})$, and of six particles on a line $(\mathbb{R})$ - and even of one particle in a 6-dimensional Euclidean space $\left(\mathbb{R}^{6}\right)$. Moreover, all these Hilbert-spaces are also isomorphic to $L^{2}(\mathbb{R})$, pertaining to a single particle on a line. Moral: to specify a Hilbert-space, which represents the set of all possible physical states of a physical system, does next to nothing to specify that physical system.

The $\otimes$-factorisation of spin and space in (24) does not mean that spin and space also $\otimes$-factorise in every state. But in the case of the anti-symmetric subspace they do. Here is again the space-spin singlet (7), in degree-of-freedom-factorised state:

$$
|\Psi(t)\rangle=\frac{1}{\sqrt{2}}(|L ; t\rangle \otimes|R ; t\rangle+|R ; t\rangle \otimes|L ; t\rangle) \otimes \frac{1}{\sqrt{2}}\left(\left|\uparrow_{z}\right\rangle \otimes\left|\downarrow_{z}\right\rangle-\left|\downarrow_{z}\right\rangle \otimes\left|\uparrow_{z}\right\rangle\right) .
$$


A vector slightly deviating from anti-symmetry, and therefore not a member of the antisymmetric state-space, is the following one in snapshot Hilbert-space, which deviates slightly from $|\Psi(t)\rangle(20)$ :

$$
\sqrt{0.49}\left(\left|\uparrow_{z} ; t\right\rangle_{1} \otimes\left|\downarrow_{z} ; t\right\rangle_{\mathrm{r}}\right)-\sqrt{0.51}\left(\left|\downarrow_{z} ; t\right\rangle_{1} \otimes\left|\uparrow_{z} ; t\right\rangle_{\mathrm{r}}\right) .
$$

This vector represents a state of two dissimilar particles. The vector does not $\otimes$-factorise in a spin and a spatial part. In the grand Hilbert-space (24), this vector looks as follows:

$$
\begin{aligned}
& \sqrt{0.49}\left|L, \uparrow_{z} ; t\right\rangle \otimes\left|R, \downarrow_{z} ; t\right\rangle-\sqrt{0.51}\left|L, \downarrow_{z} ; t\right\rangle \otimes\left|R, \uparrow_{z} ; t\right\rangle \\
& -\sqrt{0.49}\left|R, \downarrow_{z} ; t\right\rangle \otimes\left|L, \uparrow_{z} ; t\right\rangle+\sqrt{0.51}\left|R, \uparrow_{z} ; t\right\rangle \otimes\left|L, \downarrow_{z} ; t\right\rangle .
\end{aligned}
$$

Hence no snapshot Hilbert-space for dissimilar particles ensues-which is not bad news for Factorists because for dissimilar particles, even $\forall$-Factorism rules.

\section{Measurement and Locality}

Friebe (2014) considers the tangled state $|\Theta(t)\rangle(7)$. Bob performs a joint measurememt on one particle of $y$-spin and of a projector in the spectral resolution of the position-operator, that projects on the volume in space occupied by the $y$-spin detector of Bob $\left(V_{R} \subset \mathbb{R}^{3}\right)$. (This joint measurement of spin and position is possible because the corresponding operators commute.) Alice does not perform any measurement. The symmetric operator that represents Bob's measurement is this one:

$$
\frac{1}{2}\left(P^{\mathbf{Q}}\left(V_{R}\right) \sigma_{y} \otimes 1+1 \otimes P^{\mathbf{Q}}\left(V_{R}\right) \sigma_{y}\right)
$$

We further assume that Bob measures at a time $t=\tau$ when the bulk of the position-propability of $|R ; \tau\rangle$ is at $V_{R}$, so that $P^{\mathbf{Q}}|R ; \tau\rangle \approx|R ; \tau\rangle$, which implies that the probability to detect one particle at $t=\tau$ at $V_{R}$ is nearly 1 . Since the eigenstate of $y$-spin down is a superposition of eigenstates of $z$-spin up and $z$-spin down, the probability to find $y$-spin down at $V_{R}$ equals $1 / 2$.

Suppose Bob finds $y$-spin down and detects one particle at $V_{R}$. State $|\Theta(t)\rangle$ then collapses to:

$$
|\Theta(\tau)\rangle=\frac{1}{\sqrt{2}}\left(\left|L, \uparrow_{z} ; \tau\right\rangle \otimes\left|R, \downarrow_{y} ; \tau\right\rangle-\left|R, \downarrow_{y} ; \tau\right\rangle \otimes\left|L, \uparrow_{z} ; \tau\right\rangle\right),
$$

where $\left|R, \downarrow_{y} ; \tau\right\rangle$ is the Pauli-spinor localised at $V_{R}$ and being an eigenvector of $y$-spin with eigenvalue -1 . The collapsed state $|\Theta(\tau)\rangle(30)$ remains a tangled state. There was a particle Left- $z \mathrm{Up}$ before the measurement and there remains one after the measurement. The particle Right- $z$ Down, on which Bob has performed the measurement, is now Right- $y$ Down.

Next Friebe points out that if Factorism is correct (and Part States is assumed), then the post-measurement states of both particles are the partial traces of (30), and they are the same. Furthermore, their state is different from their pre-measurement state. The change of state of one particle is due to Bob's measurement of $y$-spin and location in $V_{R}$ of this 
particle (labeled ' 1 ' or ' 2 ' but one cannot know which), whereas Alice has left the other particle alone. Then why has its state changed? In case of an entangled state, we would, versed in QM as we are, have expected this, but $|\Theta(\tau)\rangle$ is a tangled state. ${ }^{26}$

Give up direct reference, and thereby Direct Factorism, embrace Ghirardi's interpretation of tangled states, which entails giving up Descriptive Factorism too, and the problem evaporates.

When we move to snapshot Hilbert-space (see Sect. 3), the tangled state $|\Theta(\tau)\rangle(30) \otimes$ -factorises and the labels of the $\otimes$-factor Hilbert-spaces can be provided with descriptions. Descriptive Factorism with specified snapshot Hilbert-space has been vindicated, and this proves $\exists$-Factorism.

\section{Concluding Remarks}

When we enter the noisy movie theatre of the grand Hilbert-space with its infinitely many dimensions (14), (24), Factorism plays the part of the whipping boy in the movie Composite Physical Systems of Similar Particles, for the tangled as well as the entangled cases. But when we watch the Schrödinger-movie in a continuous sequence of snap-shot Hilbertsubspaces of four cosy dimensions, Factorism is the indomitable hero: the labels of the Q-factor Hilbert-spaces of snapshot Hilbert-space can be taken to play descriptive roles. Descriptive reference and $\exists$-Factorism vindicated. In both Hilbert-spaces we can calculate the relevant probabilities of the position and spin degrees of freedom.

Finally, after having kissed Factorism goodbye, Caulton (2012; 2018a, b) devised the following construction. For the sake of simplicity, we stick to a composite system of two similar particles, with Hilbert-space

$$
\mathcal{H}_{ \pm}^{2}=(\mathcal{H} \otimes \mathcal{H})_{ \pm}
$$

Let $E_{\alpha}, E_{\beta} \in \mathcal{L}(\mathcal{H})$ be two orthogonal projectors (members of the lattice), which will individuate the two particles- thus the particles will become absolutely discernible. Consider the following subspace:

$$
\mathcal{H}_{ \pm}(\alpha, \beta) \equiv\left(E_{\alpha} \otimes E_{\beta}+E_{\beta} \otimes E_{\alpha}\right)\left[\mathcal{H}_{ \pm}^{2}\right] \sqsubset \mathcal{H}_{ \pm}^{2},
$$

obtained by letting a symmetric operator act on $\mathcal{H}_{ \pm}^{2}$, thus guaranteeing that its range lies inside $\mathcal{H}_{ \pm}^{2}$. One particle always has a state in subspace $\mathcal{H}_{\alpha} \equiv E_{\alpha}[\mathcal{H}] \sqsubset \mathcal{H}$ and the other particle $\mathcal{H}_{\beta} \equiv E_{\beta}[\mathcal{H}] \sqsubset \mathcal{H}$.

The state of the composite two-particle system then is in $\mathcal{H}_{\alpha} \otimes \mathcal{H}_{\beta}$. The labels of these $\otimes$-factor Hilbert-spaces then can be taken as descriptive names of the particles: the particle with state in $\mathcal{H}_{\alpha}$ and the particle with state in $\mathcal{H}_{\beta}$. Caulton ends up with nothing less than two absolutely discernible particles - and even Leibniz's Principle of the Identity of Absolute Indiscernibles is vindicated uno tenore.

Ironically, Caulton began by rejecting Factorism (in fact $\forall$-Factorism) and intended to devise an 'anti-factorist' alternative $\otimes$-factorisation of the Hilbert-space of the composite system, only to end up with a rigorous proof of $\exists$-Factorism and a refutation of Anti-Factorism. Smile, please.

${ }^{26}$ To aggravate the problem, one can easily argue that we have a breach of EPR-Locality here. Exercise. 


\section{Envoi}

We baptised non-factorisable states that result from anti-symmetrising a product-state as tangled (p. 8). Ghirardi et al. claimed that only entangled states lead to violations of Bell's inequality, and thus that tangled states do not. ${ }^{27}$ Yet take this product state:

$$
\frac{1}{\sqrt{2}}(|L, \uparrow ; t\rangle+|R, \uparrow ; t\rangle) \otimes \frac{1}{\sqrt{2}}(|L, \downarrow ; t\rangle+|R, \downarrow ; t\rangle) .
$$

Anti-symmetrising this state yields:

$$
\begin{aligned}
& |\Xi(t)\rangle=\frac{1}{\sqrt{2}}(|L ; t\rangle \otimes|L ; t\rangle+|R ; t\rangle \otimes|R ; t\rangle+|L ; t\rangle \otimes|R ; t\rangle \\
& \quad+|R ; t\rangle \otimes|L ; t\rangle) \frac{1}{\sqrt{2}}\left(\left|\uparrow_{z}\right\rangle \otimes\left|\downarrow_{z}\right\rangle-\left|\downarrow_{z}\right\rangle \otimes\left|\uparrow_{z}\right\rangle\right) .
\end{aligned}
$$

This state then must be tangled, not entangled, according to Ghirardi et al., and therefore should not violate any Bell inquality. But $|\Xi(t)\rangle$ (34) does violate a Bell inequality and is very entangled. Were Ghirardi et al. mistaken to propound that, contra communis opinio, tangled states do not deserve to be called 'entangled' because they do not lead to a violation of some Bell inequality?

Acknowledgements Thnx to Dennis Dieks, Tomasz Bigaj, Cord Friebe, Ronnie Hermens, Andrea Lubberding and two anonymous Referees for comments and suggestions.

Open Access This article is licensed under a Creative Commons Attribution 4.0 International License, which permits use, sharing, adaptation, distribution and reproduction in any medium or format, as long as you give appropriate credit to the original author(s) and the source, provide a link to the Creative Commons licence, and indicate if changes were made. The images or other third party material in this article are included in the article's Creative Commons licence, unless indicated otherwise in a credit line to the material. If material is not included in the article's Creative Commons licence and your intended use is not permitted by statutory regulation or exceeds the permitted use, you will need to obtain permission directly from the copyright holder. To view a copy of this licence, visit http://creativecommons.org/licenses/by/4.0/.

\section{References}

Bigaj, T. (2015a). Dissecting weak discernibility of quanta. Studies in History and Philosophy of Modern Physics, 50, 43-53.

Bigaj, T. (2015b). Exchanging quantum particles. Philosophia Scientae, 19(1), 185-198.

Butterfield, J. (1993). Interpretation and identity in quantum theory. Studies in History and Philosophy of Science, 24, 443-476.

Caulton, A. (2012). Qualitatively individuating so-called indistinguishable quantum systems. Talk at Cambridge University, 27 April 2012.

Caulton, A. (2013). Discerning indistinguishable quantum systems. Philosophy of Science, 80, 49-72.

Caulton, A. (2018a). Physical Entanglement in Permutation-Invariant Quantum Mechanics, LaTeX-script.

Caulton, A. (2018b). Permutations, unfinished LaTeXscript of some monograph in statu nascendi.

Cohen-Tannoudji, C., Diu, B., \& Laloë, F. (1978). Quantum mechanics (Vol. 2). New York: Wiley.

Dieks, D., \& Lubberdink, A. (2011). How classical particles emerge from the quantum world. Foundations of Physics, 41, 1051-1064.

French, S., \& Krause, D. (2006). Identity and physics: A historical, philosophical and formal analysis. Oxford: Clarendon Press.

${ }^{27}$ Ghirardi et al. (2002), Ghirardi and Marinatto (2004, 12109: 3). 
French, S., \& Redhead, M. (1988). Quantum physics and the identity of indiscernibles. British Journal for the Philosophy of Science, 39, 233-246.

Friebe, C. (2014). Individuality, distinguishability and (non-)entanglement: A defense of Leibniz's principle. Studies in History and Philosophy of Modern Physics, 48, 89-98.

Ghirardi, G., \& Marinatto, L. (2004). General criterion for the entanglement of two indistinguishable particles. Physical Review, A70(12109), 1-10.

Ghirardi, G., Marinatto, L., \& Weber, T. (2002). Entanglement and properties of composite quantum systems: A conceptual and mathematical analysis. Journal of Statistical Physics, 108(112), 49-122.

Gisin, N. (1991). Bell's inequality holds for all non-product states. Physical Letters A, 154, 201.

Hawley, K. (2009). Identity and indiscernibility. Mind, 118, 101-119.

Huggett, N., \& Imbo, T. (2009). Indistinguishability. In Compendium of quantum physics (pp. 311-317). Berlin/New York: Springer.

Huggett, N., \& Norton, J. (2014). Weak discernibility for quanta, the right way. British Journal for the Philosophy of Science, 65, 39-58.

Ladyman, J., Linnebo, O., \& Bigaj, T. (2013). Entanglement and non-factorizability. Studies in History and Philosophy of Modern Physics, 43, 215-221.

Ladyman, J., Linnebo, O., \& Pettigrew, R. (2012). Identity and discernibility in philosophy and logic. The Review of Symbolic Logic, 5, 162-186.

Lubberdink, A. (1998). De individualiseerbaarheid van van identieke deeltjes. Masters Thesis, Utrecht University.

Muller, F. A. (2015). The rise of relationals. Mind, 124, 201-237.

Muller, F. A., \& Saunders, S. (2008). Discerning fermions. British Journal for the Philosophy of Science, $59,499-548$.

Muller, F. A., \& Seevinck, M. P. (2009). Discerning elementary particles. Philosophy of Science, 76, 179-200.

Redhead, M., \& Teller, P. (1992). Particle labels and the theory of indistinguishable particles in quantum mechanics. British Journal for the Philosophy of Science, 43, 201-218.

Saunders, S. (2006a). Are quantum particles objects? Analysis, 66, 52-63.

Saunders, S. (2006b). On the explanation for quantum statistics. Studies in the History and Philosophy of Modern Physics, 37, 192-211.

Saunders, S. (2013). Indistinguishability. In R. Batterman (Ed.), Oxford handbook of philosophy of physics (pp. 340-380). Oxford: Oxford University Press.

van Fraassen, B. C. (1991). Quantum mechanics: An empiricist view. Oxford: Oxford University Press.

Publisher's Note Springer Nature remains neutral with regard to jurisdictional claims in published maps and institutional affiliations. 